\title{
Stanley depth of squarefree Veronese ideals
}

\author{
Mircea Cimpoeaş
}

\begin{abstract}
We compute the Stanley depth for the quotient ring of a square free Veronese ideal and we give some bounds for the Stanley depth of a square free Veronese ideal. In particular, it follows that both satisfy the Stanley's conjecture.
\end{abstract}

\section{Introduction}

Let $K$ be a field and $S=K\left[x_{1}, \ldots, x_{n}\right]$ the polynomial ring over $K$. Let $M$ be a $\mathbb{Z}^{n}$-graded $S$-module. A Stanley decomposition of $M$ is a direct sum $\mathcal{D}: M=\bigoplus_{i=1}^{r} m_{i} K\left[Z_{i}\right]$ as $K$-vector space, where $m_{i} \in M, Z_{i} \subset\left\{x_{1}, \ldots, x_{n}\right\}$ such that $m_{i} K\left[Z_{i}\right]$ is a free $K\left[Z_{i}\right]$-module. We define $\operatorname{sdepth}(\mathcal{D})=\min _{i=1}^{r}\left|Z_{i}\right|$ and $\operatorname{sdepth}(M)=\max \{\operatorname{sdepth}(M) \mid \mathcal{D}$ is a Stanley decomposition of $M\}$. The number $\operatorname{sdepth}(M)$ is called the Stanley depth of $M$. Stanley conjecture [1] says that $\operatorname{sdepth}(M) \geq \operatorname{depth}(M)$.

Herzog, Vladoiu and Zheng show in [5] that $\operatorname{sdepth}(M)$ can be computed in a finite number of steps if $M=I / J$, where $J \subset I \subset S$ are monomial ideals. There are two important particular cases, $I$ and $S / I$. The Stanley conjecture for $S / I$ and $I$ was proved for $n \leq 5$ and in other special cases, but it remains open in the general case. See for example, [6]. Also, the explicit computation of the Stanley depth turns out to be a difficult problem, even for simpler monomial ideals, or quotient of monomial ideals. See for instance

Key Words: Stanley depth, Stanley conjecture, Monomial ideal.

2010 Mathematics Subject Classification: Primary: 13H10; Secondary: 13P10.

Received: April, 2012.

Revised: October, 2012.

Accepted: February, 2013. 
[2], where the authors compute the Stanley depth for the monomial maximal ideal $\left(x_{1}, \ldots, x_{n}\right) \subset S$.

For any $d \in[n]$, we denote $I_{n, d}:=(u \in S$ square free monomial : $\operatorname{deg}(u)=$ d). It is well known that $\operatorname{dim}\left(S / I_{n, d}\right)=\operatorname{depth}\left(S / I_{n, d}\right)=d-1$. Let $\mathbf{m}=$ $\left(x_{1}, \ldots, x_{n}\right) \subset S=K\left[x_{1}, \ldots, x_{n}\right]$ be the maximal monomial ideal of $S$. We showed in [4] that $\operatorname{sdepth}\left(\mathbf{m}^{k}\right) \leq\left\lceil\frac{n}{k+1}\right\rceil$, for any positive integer $k$. In this paper, we use similar techniques to give an upper bound for $\operatorname{sdepth}\left(I_{n, d}\right)$. More precisely, we show that $\operatorname{sdepth}\left(S / I_{n, d}\right)=d-1$ and $d \geq \operatorname{sdepth}\left(I_{n, d}\right) \frac{n-d}{d+1}+d$, see Theorem 1.1. As a consequence, it follows that $I_{n, d}$ and $S / I_{n, d}$ satisfy the Stanley conjecture, see Corollary 1.2. Also, we prove that $\operatorname{sdepth}\left(I_{n, d}\right)=$ $d+1$, if $2 d+1 \leq n \leq 3 d$, see Corollary 1.5. In order to do so, we use some combinatorics results, see Theorem 1.3 and Corollary 1.4. Finally, we conjecture that $\operatorname{sdepth}\left(I_{n, d}\right)=\left\lfloor\frac{n-d}{d+1}\right\rfloor+d$.

\section{Main results}

Theorem 1.1. (1) $\operatorname{sdepth}\left(S / I_{n, d}\right)=d-1$.

(2) $d \leq \operatorname{sdepth}\left(I_{n, d}\right) \leq \frac{n-d}{d+1}+d$.

Proof. (1) Firstly, note that $\operatorname{sdepth}\left(S / I_{n, d}\right) \leq d-1=\operatorname{dim}\left(S / I_{n, d}\right)$. We use induction on $n$ and $d$. If $n=1$, there is nothing to prove. If $d=1$, it follows that $I_{n, 1}=\left(x_{1}, \ldots, x_{n}\right)$ and thus $\operatorname{sdepth}\left(S / I_{n, 1}\right)=0$, as required. If $d=n$, it follows that $I_{n, n}=\left(x_{1} \cdots x_{n}\right)$ and therefore $\operatorname{sdepth}\left(S / I_{n, n}\right)=n-1$, as required. Now, assume $n>1$ and $1<d<n$. Note that

$$
S / I_{n, d}=\bigoplus_{|\operatorname{supp}(u)|<d} u \cdot K=\sum_{Z \subset\left\{x_{1}, \ldots, x_{n}\right\},|Z|=d-1} K[Z] .
$$

We denote $S^{\prime}=K\left[x_{1}, \ldots, x_{n-1}\right]$. By previous equality, we get

$$
\begin{gathered}
S / I_{n, d}=\sum_{Z \subset\left\{x_{1}, \ldots, x_{n-1}\right\},|Z|=d-1} K[Z] \oplus x_{n}\left(\sum_{Z \subset\left\{x_{1}, \ldots, x_{n-1}\right\},|Z|=d-1} K[Z]\right)\left[x_{n}\right]= \\
=S^{\prime} / I_{n-1, d} \oplus x_{n}\left(S^{\prime} / I_{n-1, d-1}\right)\left[x_{n}\right] .
\end{gathered}
$$

By induction hypothesis, it follows that $\operatorname{sdepth}\left(S / I_{n, d}\right)=d-1$.

(2) We consider the following simplicial complex, associated to $I_{n, d}$,

$$
\Delta_{n, d}:=\left\{\operatorname{supp}(u): u \in I_{n, d} \text { monomial }\right\} .
$$

\footnotetext{
${ }^{*}$ The support from grant ID-PCE-2011-1023 of Romanian Ministry of Education, Re-
} search and Innovation is gratefully acknowledged. 
Note that, by [5, Theorem 2.4], there exists a partition of $\Delta_{n, d}=\bigcup_{i=1}^{r}\left[F_{i}, G_{i}\right]$, such that $\min _{i=1}^{r}\left|G_{i}\right|=\operatorname{sdepth}\left(I_{n, d}\right):=s$. Note that $\Delta_{n, d}=\{F \subset[n]:|F| \geq$ $d\}$. It follows that $\operatorname{sdepth}\left(I_{n, d}\right) \geq d$.

We consider an interval $\left[F_{i}, G_{i}\right]$ with $\left|F_{i}\right|=d$. Since $\left|G_{i}\right| \geq s$, it follows that there exists at least $(s-d)$ distinct sets in $\left[F_{i}, G_{i}\right]$ of cardinality $d+1$. Since $\Delta_{n, d}=\bigcup_{i=1}^{r}\left[F_{i}, G_{i}\right]$ is a partition, it follows that $\left(\begin{array}{c}n \\ d+1\end{array}\right)=\frac{n-d}{d+1}\left(\begin{array}{l}n \\ d\end{array}\right) \geq(s-d)\left(\begin{array}{l}n \\ d\end{array}\right)$. Thus, $s \leq d+\frac{n-d}{d+1}$.

Corollary 1.2. $I_{n, d}$ and $S / I_{n, d}$ satisfy the Stanley's conjecture. Also,

$$
\operatorname{sdepth}\left(I_{n, d}\right) \geq \operatorname{sdepth}\left(S / I_{n, d}\right)+1 .
$$

Let $k \leq n$ be two positive integers. We denote $A_{n, k}=\{F \subset[n]|| F \mid=k\}$. We present the following well known result from combinatorics. In order of completeness, we give also a sketch of the proof.

Theorem 1.3. For any positive integers $d \leq n$ such that $d \leq n / 2$, there exists a bijective map $\Phi_{n, d}: A_{n, d} \rightarrow A_{n, d}$, such that $\Phi_{n, d}(F) \cap F=\emptyset$ for any $F \in A_{n, d}$.

Proof. We use induction on $n$ and $d$. If $n \leq 2$ the statement is obvious. If $d=1$, for any $i \in[n]$, we define $\Phi_{n, 1}(\{i\})=\{j\}$, where $j=\max ([n] \backslash$ $\left.\left\{\Phi_{n, 1}(\{1\}), \ldots, \Phi_{n, 1}(\{i-1\})\right\}\right) . \Phi_{n, 1}$ is well defined and satisfy the required conditions. Now, assume $n \geq 3$ and $d \geq 2$. If $n=2 d$ we define $\Phi_{n, d}(F)=$ $[n] \backslash F$. Obviously, $\Phi_{n, d}$ satisfy the required conditions. Thus, we may also assume $d<n / 2$.

On $A_{n, d}$, we consider the lexicographic order, recursively defined by $F<G$ if and only if $\max \{F\}<\max \{G\}$ or $\max \{F\}=\max \{G\}=k$ and $F \backslash\{k\}<$ $G \backslash\{k\}$ on $A_{n, d-1}$. For any $F \in A_{n, d}$, we define $G:=\Phi_{n, d}(F)$ to be the maximum set, with respect to " $<$ ", such that $G \cap F=\emptyset$ and $G \neq \Phi_{n, d}(H)$ for all $H<F$. In order to complete the proof, it is enough to show that each collection of sets

$$
\mathcal{M}_{F}^{n}=\left\{G \subset[n]:|G|=d, G \cap F=\emptyset, G \neq \Phi_{n, d}(H)(\forall) H<F\right\}
$$

is nonempty, for all $F \subset[n]$. Assume there exists some $F \subset[n-1]$ such that $\mathcal{M}_{F}^{n}=\emptyset$. It obviously follows that $M_{F}^{n-1}=\emptyset$ and thus $\Phi_{n-1, d}$ is not well defined, a contradiction. Also, if $M_{F}=\emptyset$ for some $F \subset[n]$ with $n \in F$, it follows similarly that $\Phi_{n-1, d-1}$ is not well defined, again a contradiction. Therefore, the required conclusion follows.

Corollary 1.4. For any positive integers $d$ and $n$ such that $d<n / 2$, there exists an injective map $\Psi_{n, d}: A_{n, d} \rightarrow A_{n, d+1}$, such that $F \subset \Psi_{n, d}(F)$ for any $F \in A_{n, d}$. 
Proof. We use induction on $n$. If $n \leq 2$ there is nothing to prove. If $d=1$, we define $\Psi_{n, 1}: A_{n, 1} \rightarrow A_{n, 2}$ by $\Psi_{n, 1}(\{1\})=\{1,2\}, \ldots, \Psi_{n, 1}(\{n-1\})=\{n-1, n\}$ and $\Psi_{n, 1}(\{n\})=\{1, n\}$. Now, assume $n \geq 3$ and $d \geq 2$. If $n=2 d+1$, we consider the bijective map $\Phi_{n, d}: A_{n, d} \rightarrow A_{n, d}$ such that $\phi(F) \cap F=\emptyset$ for all $F \in A_{n, d}$ and we define $\Psi_{n, d}(F):=[n] \backslash \Phi_{n, d}(F)$. The map $\Psi_{n, d}$ satisfies the required condition.

If $n<2 d+1$, we define $\Psi_{n, d}(F):=\Psi_{n-1, d}(F)$ if $F \subset[n-1]$ and $\Psi_{n, d}(F):=$ $\Psi_{n-1, d-1}(F \backslash\{n\}) \cup\{n\}$ if $n \in F$. Note that both $\Psi_{n-1, d}$ and $\Psi_{n-1, d-1}$ are well defined and injective by induction hypothesis, since $n-1 \leq 2 d+1$. It follows that $\Psi_{n, d}$ is well defined and injective, as required.

Corollary 1.5. Let $n, d$ be two positive integers such that $2 d+1 \leq n \leq 3 d$. Then $\operatorname{sdepth}\left(I_{n, d}\right)=d+1$.

Proof. As in the proof of 1.1, we denote

$$
\Delta_{n, d}:=\left\{\operatorname{supp}(u): u \in I_{n, d} \text { monomial }\right\}=\{F \subset[n]:|F| \geq d\} .
$$

We consider the following partition of $\Delta_{n, d}$ :

$$
\Delta_{n, d}=\bigcup_{|F|=d}\left[F, \Psi_{n, d}(F)\right] \cup \bigcup_{|F|>d+1}[F, F],
$$

where $\Psi_{n, d}$ is given by the previous corollary. It follows that $\operatorname{sdepth}\left(I_{n, d}\right) \geq$ $d+1$. On the other hand, by 1.1, $\operatorname{sdepth}\left(I_{n, d}\right) \leq d+1$ and thus $\operatorname{sdepth}\left(I_{n, d}\right)=$ $d+1$, as required.

We conclude this paper with the following conjecture.

Conjecture 1.6. For any positive integers $d \leq n$ such that $d \leq n / 2$, we have

$$
\operatorname{sdepth}\left(I_{n, d}\right)=\left\lfloor\frac{n-d}{d+1}\right\rfloor+d
$$

\section{References}

[1] J.Apel, On a conjecture of R.P.Stanley, Journal of Algebraic Combinatorics, 17(2003), 36-59.

[2] C. Biro, D. M. Howard, M. T. Keller, W. T. Trotter, S. J. Young, Partitioning subset lattices into intervals, preliminary version, Preprint 2008.

[3] M. Cimpoeas, Stanley depth for monomial complete intersection, Bull. Math. Soc. Sc. Math. Roumanie 51(99)(2008), 205-211. 
[4] M. Cimpoeas, Some remarks on the Stanley depth for multigraded modules, Le Matematiche, 53(2008), 165-171.

[5] J. Herzog, M. Vladoiu, X. Zheng, How to compute the Stanley depth of a monomial ideal, Journal of Algebra, 322(2009), 3151-3169.

[6] D. Popescu, Stanley depth of multigraded modules, Journal of Algebra, 321(2008), 2782-2797.

Mircea Cimpoeas,

Simion Stoilow Institute of Mathematics,

Research unit 5, P.O.Box 1-764, Bucharest 014700, Romania

E-mail: mircea.cimpoeas@imar.ro 
